\title{
Editorial
}

Psychotherapy and Psychosomatics
Psychother Psychosom 2016;85:327-336

DOI: $10.1159 / 000448788$
Received: July 31, 2016

Accepted: August 1, 2016

Published online: October 15, 2016

\section{Clinical Factor 2015}

\author{
Richard Balon \\ Department of Psychiatry and Behavioral Neurosciences and Department of Anesthesiology, \\ Wayne State University School of Medicine, Detroit, Mich., USA
}

As in the previous 5 years [1-5], I am presenting my subjective selection, this time of 16 (primary) articles actually their summaries - published in the previous year (2015) that I believe may provide some information to help the reader improve his/her clinical practice. As I noted before, the idea was originally proposed by Giovanni Fava [6] with the goal of selecting papers that I would subjectively perceive as having a high 'Clinical Factor' the degree and extent to which a journal article provides information to the clinician that may improve his/her practice. And again, as I wrote before about the selected articles, 'many may find them useful, some will find them less useful or even useless. I am certainly biased, as is anybody else. I also cannot review all psychiatric journals - I focused only on most of the major ones. If there is no article selected from a certain major psychiatric journal, it does not necessarily mean that I have not reviewed it or skimmed through that journal. It may mean that I did not find, in my opinion, anything clinically very interesting' [1]. I view myself first and foremost as a clinician (though academically oriented) and educator, and I am, as most clinicians are, looking for a way to help patients and for guidance in the treatment of my patients, and thus looking for new, innovative, or newly validated treatments. I am sure I missed a number of valuable articles, but as noted, this is a subjective selection.

\section{KARGER}

E-Mail karger@karger.com

www.karger.com/pps

\section{Cannabis and Its Impact on Mental IIIness}

A number of studies have suggested an association between cannabis use and schizophrenia. However, the causality of this relationship is not clear - during the prodromal stage of schizophrenia individuals may suffer from a variety of nonspecific symptoms for which they may use cannabis either as a result of the illness or to treat these symptoms. Given the strong familial/genetic contributions to both schizophrenia and cannabis use/misuse, Giordano et al. [7] examined whether some or all of the association between cannabis use and schizophrenia arises from familial confounding. They tested the etiological hypothesis that cannabis is one direct cause of schizophrenia using a correlative case-control design with fullsibling, half-sibling and first-cousin comparisons, along a general Swedish population sample. Their study linked data from multiple Swedish nationwide registries and health care data, using the unique individual Swedish 10-digit personal ID number assigned to all Swedish residents. Using ICD codes, they were able to match 5,456 individuals with an initial diagnosis of schizophrenia (diagnosed between 2000 and 2010, aged under 50 at the time of the initial diagnosis) with 5 schizophrenia-free controls each (matched for gender and country of birth). They further identified first-cousin, half-sibling and full- (c) 2016 S. Karger AG, Basel

0033-3190/16/0856-0327\$39.50/0
Richard Balon, MD

Departments of Psychiatry and Behavioral Neurosciences and Anesthesiology Tolan Park Building

Third Floor, 3901 Chrysler Service Drive, Detroit, MI 48201 (USA)

E-Mail rbalon@wayne.edu 
sibling pairs discordant for cannabis abuse and statistically extrapolated the results for discordant monozygotic twins. From all schizophrenia cases derived from the Swedish general population, $10.28 \%$ were recorded as cannabis abusers prior to diagnosis, compared with $1.17 \%$ among controls. There was a large increased risk of schizophrenia if an individual had a prior registration of cannabis abuse [odds ratio $(\mathrm{OR})=10.44,95 \%$ confidence interval $(\mathrm{CI})=8.99-12.11]$. This association was substantially attenuated both by increasing temporal delays between cannabis abuse and schizophrenia diagnosis and by controlling for increasing degrees of familial confounding (the risk remained substantial - almost twofold - and significant at 7 years, $\mathrm{OR}=4.24,95 \% \mathrm{CI}=$ 3.54-5.07). Interestingly, opiate, sedative, cocaine/stimulant, and hallucinogen abuses were also strongly associated with subsequent schizophrenia in the general population in this study, but after controlling for familial confounding only cocaine/stimulant exposure remained associated with a future schizophrenia risk. These results suggest that a large part of the cannabis abuse-schizophrenia association in the general population is not causal and results from confounding due to shared familial factors. Nevertheless, cannabis abuse has an appreciable causal impact on the future risk of schizophrenia. The study was limited by reliance on hospital-based diagnosis, the way cannabis abuse was diagnosed (medical and legal records), and the lack of exact information on when the abuse or illness started. The results of this study provide empirical evidence lending further support to the hypothesis that cannabis abuse is one component cause of schizophrenia. [Funding for this study was provided by the national Institute for Drug Abuse (NIDA), the Swedish Research Council, the Swedish Research Council for Health, Working Life and Welfare, and the Access to Learning Fund from the region Skane.]

Cannabis is also the most common illicit drug used by people with bipolar disorder (lifetime prevalence ranging from 26 to $46 \%$ ). Zorrilla et al. [8] studied the impact of cannabis abuse on bipolar disorder by examining whether bipolar patients who stop cannabis use during a manic/ mixed episode have a better clinical and functional outcome than those who continue to use or never used cannabis. They conducted a post hoc analysis of data from the European Mania in Bipolar Longitudinal and Evaluation of Medication study (2-year prospective observational study by 530 investigators across 14 European countries) to examine the course of bipolar disorder according to patient cannabis use habits controlling for various potential confounding variables. Their subsample of eligible patients with data on cannabis/alcohol/substance use and follow-up data included 1,922 patients - 132 (6.9\%) current cannabis users, 89 (4.6\%) previous cannabis users and 1,701 (88.5\%) never users (for comparison, alcohol: $37.7 \%$ current users, $13.4 \%$ previous users, and 49.0\% never users; other substances: $3.7 \%$ current users, $4.4 \%$ previous users, and $91.9 \%$ never users). Functional outcome measures included work impairment, relationship status, and living situation measured at the last available maintenance phase visit. Clinical outcomes included remission, recovery, recurrence, and relapse, each specifically defined (e.g. for remission by Clinical Global Impression Bipolar Disorder Scale score $<3$ at two consecutive visits and no relapse between those visits). Interestingly, previous cannabis users had the highest rates of remission (68.1\%) and recovery (38.7\%), and the lowest rates of recurrence (42.1\%) and relapse (29.8\%). Comparable rates were for current users: remission $42.6 \%$, recovery $18.9 \%$, recurrence $60.3 \%$, and relapse $46.3 \%$, and for never users: remission $61.9 \%$, recovery $31.8 \%$, recurrence $47.3 \%$, and relapse $34.2 \%$. Logistic regression showed that previous users had similar outcomes to never users (all $\mathrm{p}>0.05$ ), while current users had lower recovery ( $\mathrm{p}=$ $0.004)$ and remission $(\mathrm{p}=0.014)$, higher recurrence $(\mathrm{p}=$ $0.014)$, greater work impairment $(\mathrm{p}=0.016)$, and were more likely not to be living with a partner $(\mathrm{p}=0.006)$ than never users. The median time to remission - 571 days was longest in the current user group (never users 236 days; previous users 189 days), while the times to relapse and recurrence were shorter in the current use group. Current cannabis use during the maintenance phase was associated with lower rates of symptomatic remission and functional recovery, and a higher risk of recurrence of bipolar disorder, independent of baseline characteristics. It was also associated with poorer functional outcomes. Patients who stopped using cannabis during a manic/mixed episode had similar clinical and functional outcomes to never users. As the authors pointed out, their findings imply that stopping cannabis use is an important goal to achieve in cannabis users with bipolar disorder. The limitations of this study include relatively small numbers in groups, difficulty to accurately assess cannabis use, confounding impact of alcohol use, and post hoc character of this analysis. (This study was supported by the Health Research Funds from the Spanish Government, European Regional Development Funds and local grants as from the 'Basque Country Government' and 'University of the Basque Country'. The original EMBLEM study was supported by Eli Lilly. Several authors declared grants, consultancies or speaker's engagement with various pharma- 
ceutical companies, with significant involvement with Eli Lilly: Drs. Zorilla, Aguado, Gonzales-Pinto, Haro, LopezPena, and Lopes-Zurbano.)

As an increasing number of states in the USA have approved posttraumatic stress disorder (PTSD) as a qualifying condition for medical marijuana without much evidence of its effect on PTSD, Wilkinson et al. [9] decided to examine the association between marijuana use and PTSD symptom severity in a longitudinal observational study. They used data drawn from the national evaluation of specialized intensive PTSD programs implemented by the Northeast Program Evaluation Center of the Veterans Health Administration from 1992 to 2011. All patients entering these programs were evaluated at baseline and 4 months after discharge using a standardized set of sociodemographic and clinical measures (e.g. PTSD symptom severity, other comorbid psychiatric diagnoses, history of psychiatric hospitalization, drug and alcohol severity, chronic medical problems). Outcome measures included 4-month follow-up assessment of PTSD symptom severity, employment status, violent behavior, and composite measures of alcohol and drug use. The sample from which the subjects were selected included 47,310 veterans with a diagnosis of PTSD. Subjects with problematic alcohol use (more than 2 drinks on 1 occasion), those with any drug use other than marijuana in the 30 days prior to admission, and those who entered treatment on transfer from an inpatient or residential program that would restrict their access to alcohol or drugs were excluded to minimize any confounding effect of other drugs than marijuana. After applying those criteria, they obtained a sample of 12,770 subjects, mostly nonusers $(11,344)$. As they selected a more balanced random sample of nonusers (850), the final study population consisted of 2,276 mostly male (96.7\%) veterans (299 who stopped using marijuana during the treatment program, 296 continuous users of marijuana, 831 who started to use marijuana during the program and 850 nonusers). After adjusting for relevant baseline covariates, marijuana use was associated with worse outcome in PTSD symptom severity $(\mathrm{p}<0.01)$, violent behavior $(\mathrm{p}<0.01)$, and measures of alcohol and drug use $(\mathrm{p}<0.01)$ when compared to stoppers and never users. Importantly, at follow-up, stoppers and never users had the lowest levels of PTSD symptoms $(\mathrm{p}<0.0001)$ and starters had the highest levels of violent behavior $(\mathrm{p}<0.0001)$. The robust association of the initiation of marijuana with higher follow-up measures of violent behavior was fairly unexpected. This could be partially explained by the fact that starters had also higher rates of alcohol use at follow-up. The limita-

Clinical Factor 2015 tions of this study include lack of randomization to receive marijuana and placebo upon entering the initial treatment programs, lack of verification of drug use by toxicology, lack of assessment of varying levels of cannabinoids in the marijuana used, predominantly male gender of subjects, the fact that evaluations were done when subjects were not under the influence, and the relatively small numbers in individual groups. Nevertheless, the results have important implication for clinical practice. Marijuana may worsen PTSD symptomatology, nullify the benefits of specialized treatment and may also be associated with more violent behavior in PTSD patients. Thus, cessation and/or prevention of marijuana use in PTSD rather than the use of medical marijuana should be one of the treatment goals. (Dr. Wilkinson's work was supported in part by an NIMH grant, and he also received a grant of USD 2,500 from Janssen/APA Foundation for a project involving electroconvulsive therapy. Drs. Stefanovics and Rosenheck had no conflict of interest or funding for this study.)

Cannabis use is decreasing in England and Wales (from 10.7 to $6.6 \%$ between 2002/2003 and 2013/2014) while the demand for cannabis treatment in addiction services is still rising (e.g. from 7,579 to 11,821 adults between 2005/2006 and 2013/2014). There are now more first-time clients for cannabis treatment in Europe than for any other illicit drug. Freeman and Winstock [10] decided to examine whether this paradoxical increase is partly due to an increased availability of high-potency cannabis. Currently, cannabis products are classified into 3 broad types: (1) high potency ('skunk' - around 15\% $\delta_{9}$-tetrahydrocannabinol (THC) content; (2) low potency ('herbal', 'grass', 'weed' - around 9\% of THC), and (3) compressed block of plant matters ('resin', 'hashish' around 5\% of THC). It seems that the use of high-potency 'skunk' has been increasing. Freeman and Winstock thus conducted an online, cross-sectional drug survey in 2009. The survey collected demographic data and detailed information on the use of and experience with a number of substances. In this report they presented data on subjects in the UK only, asking separate questions about resin, skunk and grass. They compared the use of cannabis types (use in the last 12 months; days used in the last month; how long $1 / 8$ of an ounce lasts; how many joints from $1 / 8$ of ounce), profiling cannabis types (which gives the best high; which is the best value for money; which is the most likely to get you paranoid; which is the most likely to affect your memory; which is your preferred type; which is most available), routes of administration, and severity of dependence and cannabis-related 
concerns. They obtained data for 2,514 responders. In the previous year, $72.5 \%$ used skunk, $68.6 \%$ used other grass and $58.7 \%$ used resin, while $37 \%$ used actually all three preparations. Most (70.2\%) users were young males (mean age of 24.3 years). Frequent use of high-potency cannabis predicted a greater severity of dependence, and this effect became stronger as age decreased. High-potency cannabis was also clearly distinct from low-potency varieties by its marked reported effect on memory and paranoia, while reportedly providing the best high. This study had several important limitations: online survey, self-selection, cross-sectional character, possible lack of knowledge of terms such as skunk on part of the responders, and lack of using a structured interview. Nevertheless, the results suggest that more potent cannabis may be associated with a higher degree of dependence and with a higher degree of associated psychopathology. It would be important to examine whether the more potent cannabis plays a larger role in worsening of psychopathology of various mental disorders during cannabis abuse [7-9]. (Dr. Freeman's work was supported by the Medical Research Council. The study was self-funded.)

\section{Early Intervention and Prevention}

It is well known that early-starting, chronic conduct problem young children are at high risk of growing into antisocial adults and are labeled as suffering from externalizing psychopathology, or having antisocial personality disorder. They are also at risk of developing substance abuse, risky sexual behavior, harsh parenting of offspring and poor health and well-being. Thus, any early intervention that would prevent the development of these later life problems would be highly desirable. Dodge et al. [11] tested the efficacy of an early intervention program for 'early starters' - kindergarten age children with early conduct problems. Kindergarten children in three cohorts $(1991-1993, \mathrm{n}=9,954)$ at 55 schools in 4 communities (Durham, N.C.; Nashville, Tenn.; rural Pennsylvania; Seattle) in neighborhoods with high rates of crimes and economic disadvantage were screened for conduct problems, and 891 were enrolled in this study (51\% African American, 47\% European American; 69\% boys). Children were randomly assigned to 10 -year intervention or control (98\% participated during grade 1 and $80 \%$ participated through grade 10). There were different interventions offered for grades $1-5$ and $6-10$. The intervention goal was to develop social competencies through social skills training, parent behavioral-management training with home visiting, peer coaching, reading tutoring and classroom social-emotional curricula (the cost was USD 58,000 per child over the 10 years). Manualization and supervision ensured program fidelity. The authors assessed outcomes at subjects' age 25 through administrative record review and psychiatric interviews with participants and peer reporters. They focused on 8 domains: externalizing psychopathology, internalizing psychopathology, substance abuse, criminal conviction, risky sexual behavior, aggression toward partners and offspring, education/employment and well-being. Intent-to-treat logistic regression indicated that $59 \%$ of those assigned to active intervention displayed at least one externalizing, internalizing, or substance abuse problem (based on self- or peer review interview) at the age 25 compared to $69 \%$ controls (OR = $0.59, \mathrm{CI}=0.43-0.81$, number needed to treat $=8$ ). Those receiving intervention also had lower severity-weighted violent and drug crime conviction scores, lower risky sexual behavior scores and higher well-being scores. Children assigned to intervention had a significantly lower probability of meeting criteria of antisocial personality disorder and avoidant personality and a marginally significant lower probability of attention deficit hyperactivity disorder. Intervention did not affect the probability of graduating from high school or being employed full-time at the age of 25 . The main limitation of this study is that the active ingredients of the intervention are not clear and impossible to determine. The differences were also small at times and their magnitude was not clear from the report. Nevertheless, this study provides evidence that early intervention (complex, expensive) may help in preventing development of adult psychopathology in highrisk early-starting conduct problem children. (This study was funded by grants from the National Institute of Mental Health, Department of Education, and National Institute on Drug Abuse.)

As there have been calls for a combined approach to eating disorders and obesity prevention in adolescents in school setting, Wilksch et al. [12] decided to test the efficacy of three school-based programs (Media Smart and Helping, Encouraging, Listening and Protecting Peers HELPP - targeting similar eating disorder risk factors, and Life Smart targeting a wide range of both shared and obesity risk factors) in reducing eating disorder and obesity risk factors in a randomized, controlled trial comparing these three programs and a nonintervention group. They randomly allocated 1,316 grade 7 and 8 girls $(\mathrm{n}=$ $840,64 \%)$ and boys $(n=476)$ from 12 schools across three Australian states. In each school, one grade was the intervention year level (e.g. grade 7) while the other grade (e.g. 
grade 8 ) served as no-intervention control participants who would attend their usual classes. Classes in the intervention grade were randomly allocated to one of the three programs. All three programs were developed around evidence-based principles of being interactive, avoiding psychoeducation about eating disorders and obesity, and having multiple sessions ( 8 of 50 min duration at the rate of 2 lessons per week). Risk factors were measured at baseline, at 5 weeks after program, and at the 6- and 12-month follow-up. Media Smart girls had half the rate of onset of clinically significant concerns about shape and weight than control girls at the 12-month follow-up. Media Smart and HELPP girls reported a significantly lower weight and shape concern than Life Smart girls at the 12-month follow-up. Media Smart girls and boys did well on several other parameters. Media Smart was the only program to show benefit on both disordered eating and obesity risk factors. The study had a number of limitations (e.g. a relatively short follow-up period, there were some baseline differences in spite of randomization, a need for more objective measures of dieting and exercise and others). In spite of the limitations, this study suggests that some well-developed school programs may help in reducing risk factors of childhood and adolescent eating disorders and obesity (the short follow-up does not allow any speculation beyond this age group). (This research was funded by a Butterfly Research Institute Grant. S.M. Wilksch held a research fellowship funded by the South Australian Centre for Intergenerational Health. S.M. Byrne was supported by the Ellen Feldberg Gordon Fund for Eating Disorders Research and the US Maternal and Child Health Bureau.)

As Ginsburg et al. [13] pointed out, etiological models of anxiety disorders implicate several family-based risk factors. In addition, family aggregation studies indicate that children of anxious parents have an elevated risk of having an anxiety disorder, and specific parenting practices, such as modeling of anxiety and overcontrol/overprotection, contribute to elevated anxiety. Family-based therapies have been shown to reduce child anxiety, but anxiety prevention studies have not been very successful and have been done mostly in the school environment. Thus, Ginsburg et al. [13] examined the efficacy of familybased intervention to prevent the onset of anxiety disorders in offspring of anxious parents. They enrolled 136 families with a parent meeting DSM-IV criteria of an anxiety disorder and one child 6-13 years old without anxiety disorder. Families were randomly assigned to the familybased intervention ( 8 weekly 60 -min manualized sessions and 3 optional monthly booster sessions; $\mathrm{n}=70$ ) or to an information-monitoring control condition $(\mathrm{n}=66)$. All families were expected to complete assessments administered by blind interviewers, at baseline, at the end of intervention (or 8 weeks after randomization) and at 6-and 12 -month follow-ups. Onset of any anxiety disorder in a child and anxiety severity at 12 months were the primary and secondary outcome measures (simple measures!). Three children (5.26\%) in the intervention group and 19 children $(30.65 \%)$ in the control group developed an anxiety disorder $(\mathrm{OR}=8.54,95 \% \mathrm{CI}=2.27-32.06$; number needed to treat $=3.9$ ). At the 1 -year follow-up, youths in the control group also had higher anxiety symptom ratings than those in the intervention group. Significant moderators included baseline levels of child anxiety, and significant mediators were parental distress and modeling of anxiety. The limitations included a relatively small sample, participation of volunteers from predominantly Caucasian, two-parent families with relatively high incomes, the majority of parents having generalized anxiety disorder (excessive worry), the fact that the study did not control for therapist time and attention, and a relatively short follow-up of 1 year. Family therapy intervention in families of anxious parents may be useful in reducing the 1-year incidence of anxiety disorders among offspring of anxious parents. The question remains whether the intervention prevents the development of anxiety disorders or just postpones their onset. The answer to this question would require a fairly long follow-up. (This study was supported by an NIMH grant.)

Two studies examined whether cognitive-behavioral therapy (CBT) can prevent or reduce the incidence of major mental illness or its episode. Ising et al. [14] studied the cost-effectiveness and cost utility of CBT in the prevention of first-episode psychosis. In this multicenter trial, 196 ultra-high-risk (as determined by the Comprehensive Assessment of At-Risk MentalStates - CAARMS) were assigned to either routine care (101 subjects) or to routine care plus add-on individual CBT aiming at the prevention of a first episode of psychosis (95 subjects). CBT consisted of a maximum of 26 sessions with experienced CBT therapists. The outcome measures were (a) transition to psychosis as measured by CAARMS (done at $0,2,4,6,9,12,15$ and 18 months), health-related quality of life (QUALY - quality-adjusted years of life), assessment of functioning, assessment of depression, and resources use and costing (intervention costs, direct medical costs other than intervention, participants' travel costs, and costs stemming from lower productivity over 18 months). In the add-on CBT group, $10.5 \%$ of the participants made the transition to psychosis during the 
18 months (5.35\% at 6 months, at the end of the experimental period), while in the routine care group, $23.8 \%$ of the participants transitioned to psychosis during 18 months (13.9\% at 6 months). The risk difference was statistically significant $(\mathrm{p}=0.004$; number needed to treat $=7.72$ ). CBT showed a $63.7 \%$ probability of being more cost-effective, because it was less costly than routine care by USD 844 per prevented psychosis. (This study was supported by a grant from the Netherlands Organization for Health Research and Development.) In a randomized controlled trial conducted in Japan, Imamura et al. [15] investigated whether an Internet-based computerized $\mathrm{CBT}$ can decrease the risk of major depression during a 12-month follow-up among workers of 2 companies. This report is basically an extension of their previous report [16] in which they reported 6-month follow-up results. Participants included those who did not report major depression within the past month, with no lifetime diagnosis of bipolar disorder, no treatment for mental health problems within the last month, and no more than 15 sick days in the previous 3 months (all reported via Internet, after being recruited via e-mail). Participants were randomly assigned to intervention or control group (381 participants each). The intervention consisted of 6 weekly lessons and homework of Internet-based CBT (using manga - Japanese comic - story), while the control group received a short e-mail about non-CBT stress management tips, such as getting good sleep and not drinking too much, monthly for 6 months). The primary outcome measure was a new onset of major depressive episode (diagnosed by using a web version of the Composite International Diagnostic Interview). During the 12-month follow-up, $3(0.8 \%)$ participants in the intervention group and $15(3.9 \%)$ participants in the control group developed major depression (the difference was statistically significant at $\mathrm{p}<0.01$, yet the relative risk was low $-0.20-$, and the number needed to treat to achieve the prevention of 1 case of onset of major depression was 32 ). This study has a number of limitations diagnoses were made by self-report, not everybody finished all sessions, participants were mostly educated male professionals working for IT companies. Nevertheless, this study demonstrates the possible usefulness of Internet-based CBT in work places. [This study was supported by grants from the Japan Society for the Promotion of Science. The authors received (individually) various honoraria from various pharmaceutical companies, and the first author is employed part-time by Chugai Pharmaceutical Company and Medical Care Toranomon.]
Two studies investigated the prevention of suicidal ideation $[17,18]$ and suicidal behavior [18]. It is well known that physicians, especially physicians in training, are at high risk for suicide compared with general population. Despite increased suicidal ideation and other mental problems among interns, very few of them seek help/treatment. Lack of time, preference to manage problems on their own, lack of convenient access to care and concerns about confidentiality have been cited as barriers to treatment of mental health issues among physicians. Guille et al. [17] thus tested the feasibility, acceptability, and effectiveness of a web-based CBT (wCBT) program for the reduction of suicidal thoughts among medical interns. Webbased therapies offer enhanced confidentiality, low cost, easy accessibility, flexibility in timing of access and selfmanagement tools. Guille et al. enrolled interns in various medical specialties from two US university hospitals (Yale University and University of Southern California) and randomly assigned them to wCBT (4 weekly web-based sessions lasting $30 \mathrm{~min}$ each) or attention-control condition (4 weekly e-mails about the prevalence of depression and suicide among physicians, symptoms of depression and suicide and encouragement to seek help, locally, if necessary, with contacts provided). The intervention group included 100 interns, 12 did not complete the modules, 19 were lost to follow-up. The control group included 99 interns, 15 were lost to follow-up. The main outcome measure was the Patient Health Questionnaire-9 which was used to assess suicidal ideation. During at least 1 point over the course of 1 year, $12 \%$ of interns in the intervention group and $21.2 \%$ of interns in the control group endorsed suicidal ideation (interestingly, the highest incidence was at 6 months). After adjusting for covariates, interns assigned to wCBT were less likely to endorse suicidal ideation during the internship year (relative risk = $0.40,95 \% \mathrm{CI}=0.17-0.91, \mathrm{p}=0.03$ ). The study limitations included self-reporting, assessment of suicidal ideation and nonsuicidal behavior, and relatively low numbers and just 2 medical centers participating. Nevertheless, this study confirms the wCBT feasibility and acceptability and possibly efficacy in the prevention of suicidal ideation among medical interns. As Reynolds [19] noted, this is the first controlled experimental observation about the efficacy of a learning-based approach to reduce the risk of suicide in physicians. (The study of Guille et al. was funded by grants from the National Institute of Child Health and Human Development, the Office of Women's Health, NIH, NIDA and American Foundation for Suicide Prevention. Dr. Reynolds' work was supported by a NIMH grant and Endowed Chair in Geriatric Psychiatry.) 
Another group with an increased risk of suicide comprises prisoners. In a relatively small pilot randomized controlled study Pratt et al. [18] examined the feasibility of cognitive-behavioral suicide prevention (CBSP) for suicidal male prisoners who had been identified as being at risk of suicidal behavior within the last month prior to the study. They assigned 31 male prisoners to CBSP and 31 male prisoners to treatment as usual (TAU). The CBSP consisted of up to 20 hourly sessions twice weekly during the initial phase of therapy, and once weekly when therapeutic relationship was established. CBSP was delivered by experienced clinical psychologists. The TAU received the usual care and support available to any prisoner. The primary outcome measure was self-injurious behavior occurring within the past 6 months (evaluations were done by research assistants at 4 months end of treatment, and at 6 months after the baseline assessment), assessed by examination of participants' prison records. The secondary outcome measures were dimensions of suicidal ideation, psychiatric symptomatology, personality dysfunction and psychological determinants of suicide, including depression and hopelessness (measured by the Beck Scale for Suicidal Ideation and the Suicide Probability Scale). The CBSP participants achieved a significantly greater reduction in suicidal behaviors with moderate treatment effects. The mean number of self-injurious behaviors was initially lower for the CBSP group $(\mathrm{CSBP}=1.06$; $\mathrm{TAU}=1.39)$; however, at 6 months the mean number of self-injurious behaviors for the CBSP group decreased by almost $50 \%$ to 0.58 , while there was little change (1.48) of these behaviors for the TAU group. Interestingly, no participant in the CBSP group had an increase in self-injurious behavior over baseline, while 6 participants in the TAU group had increases in self-injurious behavior over baseline. Significant improvements were achieved on secondary measures. While the population in this study was small and a high proportion (64\%) of eligible prisoners chose not to participate (question of feasibility), the results suggest that CBSP may be a suitable intervention for decreasing self-injurious behavior in male prisoners. (This study was funded by the National Institute for Health Research.)

\section{Use of Technology?}

As Berle et al. [20, p. 61] pointed out, 'delivery of psychological therapies via Internet holds great promise: there are benefits of accessibility, reduced stigmatiza- tion, as well as potential reduction in the cost of service provision. A large and expanding evidence base suggests that these approaches are as effective as face-to-face therapy for many disorders'. Yet in their small study (55 patients) patients overwhelmingly preferred individual face-to-face therapy to all other delivery options (group face-to-face therapy, bibliotherapy, online therapy without therapist contact and online therapy with weekly therapist phone contact). The authors noted that online therapy with weekly therapist phone contact was preferred over face-to-face group therapy and bibliotherapy. They felt that this suggests that appropriately resourced online therapy where therapists retain regular involvement with patients may be a 'favorable' next option for patients when routine individual face-to-face therapy is not available. The findings of this study were limited by the small sample, self-selection of subjects, nonvalidated survey and other factors. Nevertheless, it seems obvious that patients prefer face-to-face therapy. (There was no funding for this study and no apparent conflict of interest.)

Yet, studies examining the efficacy of therapy (mostly CBT) using various technologies keep appearing. Two studies discussed above $[15,17]$ demonstrated implementation of CBT via the web/Internet and its feasibility and acceptability in special populations or situations. Several other recent studies demonstrated efficacy of CBT delivered via modern technologies in recurrent depression [21, via mobile phone], anxiety and depression $[22$, via telemedicine], postnatal depression [23, via telephone] and generalized anxiety [24, via telephone]. One may ask whether there is a use for technology beyond delivering CBT in different variations, locations and indications. One such area is, or could be, electronic selfmonitoring of symptomatology in various disorders. As Faurholt-Jepsen et al. [25] pointed out, there has been a shift in illness paradigm in the area of mood disorders, from a focus on mood episodes in bipolar disorder to an increasing focus on interepisodic mood instability. This instability is associated with impaired global functioning and high risk of relapse. Frequent, preferably daily monitoring of interepisodic symptomatology may provide useful and important data for tailoring timely and clinically useful intervention. Face-to-face daily monitoring would be ideal, but is clearly not feasible and economically viable. Recently, a number of different types of electronic self-monitoring instruments for a variety of illnesses have been developed. As smartphones are widely disseminated and frequently used, they present a unique device for continuous and long-term monitoring of 
mood disorder symptomatology. No separate monitoring device is necessary, as numerous monitoring applications could be installed on smartphones. A number of studies documented patient adherence and acceptance of electronic self-monitoring. However, the effect of selfmonitoring in bipolar disorder has never been tested in a randomized controlled trial. Thus, Faurholt-Jepsen et al. [25] conducted a randomized, controlled, singleblind (double-blinding was impossible) trial of 6-month daily self-monitoring of depressive and manic symptomatology via smartphone in 78 patients with bipolar disorder. They assigned 39 patients to an Android smartphone-based self-monitoring system (MONARCA) and 39 patients to Android smartphone without this system (all patients also received treatment as usual). The patients assigned to the MONARCA system were prompted at a self-chosen time during the day to evaluate a number of items: mood, sleep length, medications taken, activity, irritability, mixed mood, cognitive problems, alcohol consumption, stress, menstruation in women, and individualized warning signs. The system included two levels of feedback loop, one between the patient and the clinic where the study nurse reviewed the data and contacted the patient if there were signs of deterioration and one feedback loop within the patients themselves, where the data were visualized graphically, providing the patient with an overview of entered data. Primary outcome data were Hamilton Depression Scale and Young Mania Rating Scale monthly ratings of depressive and manic symptomatology. There were also numerous secondary and tertiary outcome measures. Intention-to-treat analyses using linear mixed models showed no significant effects of daily self-monitoring using smartphones on depressive as well as manic symptomatology. The study has several important limitations, such as a small sample and well-defined and executed intervention program of medication and psychopharmacology in addition to monitoring (thus hard to detect the effect of any additional intervention). The study period - 6 months - was also relatively short. The lack of positive results in a study of intuitively useful and appealing daily monitoring of symptomatology provides a cautionary tale about introducing such technology into clinical practice. (This study was funded by: EU 7 Programme, and by Mental Health Services, Copenhagen, Denmark; Trygfonden; the Gert Einar Joergensens Foundation, and the A.P. Moellers Foundation og Hustru Chastine McKinney Moellers Foundation. Several authors served as consultants to various pharmaceutical companies not involved in this trial.)

\section{Issues in the Management of Depression}

Long-term maintenance treatment of recurrent depression reduces the risk of recurrence or relapse. Maintenance antidepressant treatment for at least 2 years is highly recommended (depends on the clinical picture and country), but as Kuyken et al. [26] pointed out, many patients are interested in alternatives to maintenance antidepressant treatment. One such alternative that has been shown to reduce risk or relapse or recurrence of depression compared with usual care is mindfulness-based cognitive therapy (MBCT). Kuyken et al. [26] decided to find out whether MBCT with support to taper or discontinue antidepressants was superior to maintenance antidepressant treatment in the prevention of depressive relapse or recurrence over 2 years. They recruited adult depressed patients with 3 or more previous depressive episodes and on a therapeutic dose of maintenance antidepressant from primary care practices in urban and rural settings in the UK. They assigned 212 patients to MBCT and 212 patients to maintenance antidepressant treatment (183 patients in each group were available for a 2-year follow-up, 212 in each group were included in the intention-to-treat population). As this was a single-blind, parallel group randomized controlled trial, patients were aware of treatment allocation, while research assessors were blind to treatment condition. MBCT is a manualized, group-based skills training designed to enable patients to learn skills that prevent the recurrence of depression (awareness of bodily sensations, thoughts and feelings associated with relapse). The MBCT program consisted of 8 sessions, $2.25 \mathrm{~h}$ each over consecutive weeks with 4 refresher sessions roughly every 3 months over the following year. Patients in the MBCT group received support to taper or discontinue their maintenance antidepressants both from their MBCT therapists and their general practitioners usually after 6 weeks of treatment. Patients in the maintenance antidepressant group received support from their general practitioners to maintain their antidepressant treatment. The primary outcome measure was time to relapse or recurrence of depression, with patients followed up at 5 separate intervals during the 2 -year period. The time to relapse or recurrence of depression did not differ between both groups, nor did the number of serious adverse events (which were not considered to be attributable to the interventions). Both treatments were associated with positive outcomes and quality of life. The authors cite the inclusion of patients with a high risk of depression (not generalizable to all types of depression) and the lack of an attention control group as limitations of this study. However, an- 
other limitation was the fact that $30 \%$ of patients in the MBCT group remained on antidepressants $(13 \%$ on the same and $17 \%$ on a reduced dose), and $24 \%$ in the antidepressant maintenance group reduced their dose. (This study was supported by the National Institute for Health Research Technology Assessment.) As Mulder [27] noted in an accompanying editorial, 'despite the increased use of drugs, the long-term outcome of mood disorders has not improved in the modern era. Having an alternative nonmedication strategy to reduce relapse is an important means to help patients with depression. (Dr. Mulder has no conflict of interest.)

Schramm et al. [28] compared Cognitive Behavioral Analysis System of Psychotherapy (CBASP) to escitalopram (plus clinical management) in patients with chronic major depression at two university clinics in Germany. CBASP is a highly structured interpersonal learning approach integrating behavioral, cognitive, and mainly interpersonal treatment strategies. Twenty-nine patients were assigned to receive 22 sessions of CBASP (first 8 weeks biweekly), and 31 patients received 18 sessions of clinical management (the escitalopram dose was usually increased to $20 \mathrm{mg} /$ day after the first week on $10 \mathrm{mg} /$ day) over 28 weeks. However, patients who had not improved at least $20 \%$ on the Montgomery-Asberg Depression Rating Scale (MADRS, the study's primary outcome measure) by the end of the 8 weeks were offered the other treatment in addition, thus creating a third group for the following 20 weeks of extended treatment. Thus, 17 patients (but 19 included in analysis) completed the 28 weeks of CBASP only, 14 those of escitalopram only, and 17 those of the combined treatment (but 20 patients included in analysis in each of these 2 groups). Depression scores decreased significantly after 8 and 28 weeks in the intent-to-treat analysis of clinicians' MADRS ratings with no significant differences between groups. The response rates after 28 weeks were $68.4 \%$ in the CBASP treatment-only group and $60.0 \%$ in the escitalopram/clinical management-only group. Remission rates were $36.8 \%$ for the CBASP group and $50.0 \%$ for the escitalopram/clinical management group (no group being superior). The combined group of initial nonimprovers achieved similar improvements at 28 weeks (larger improvement between weeks 8 and 28 than in the other two groups). Thus, for some patients the CBASP and escitalopram appeared equally effective. The combination of both treatments (basically augmenting one with the other one and vice versa) for nonresponders at 8 weeks seems to be a reasonable strategy. The study was limited by its small size, lack of placebo condition and lack of blinding of patients and therapists (common weakness of all psychother- apy studies). However, MADRS raters were blind to treatment conditions. The results of this study offer evidence for a reasonable clinical strategy for combination/augmentation of various treatment approaches. (The study was supported by Lundbeck GmbH, Hamburg.)

As Martiny et al. [29] note, many depressed patients do not reach full remission after a full trial or naturalistic study and continue to have various residual symptoms such as insomnia, lack of concentration, fatigue, sexual dysfunction, anxiety, pain and hypersomnia. Many pharmacological and nonpharmacological (not just psychotherapy) interventions have been examined in addressing these symptoms, among them chronotherapeutics and exercise. Chronotherapeutics encompass wake therapy, sleep phase advance, sleep time stabilization, and bright light therapy. Chronotherapeutic methods have been known to induce a fast and sustained response in depression, and exercise has been found to have moderate effects on depression. Martiny et al. [29] investigated the longterm antidepressant effect of chronotherapeutic intervention (combination of wake therapy, bright light therapy, sleep phase advance, and sleep time stabilization) as augmenting strategy to duloxetine in a randomized trial comparing this regime to exercise regime (30 min daily) with duloxetine daily during the initial phase of 9 weeks. This study reports the results of a 20-week follow-up after the initial intervention phase of 1 week (with an immediate large effect of chronotherapeutics compared to exercise [30]) and a continuation phase of another 7 weeks (again, showing a large difference between chronotherapeutics and exercise [31]). Patients continued to use bright light therapy and sleep time stabilization and were able to change medication during this follow-up phase. Patients were assessed every 4 weeks. Remission rates were the primary outcome. Patients in the chronotherapeutics groups (37 subjects; originally received wake therapy and other chronotherapeutics, bright light and sleep time stabilization were continued in the follow-up phase) had a significantly higher remission rate of 61.9 versus $37.9 \%$ in the exercise group of 38 patients at week $29(\mathrm{OR}=2.6, \mathrm{CI}=$ $1.3-5.6, \mathrm{p}=0.01)$. Sleep maintenance was significantly better in the chronotherapeutics group. These results demonstrated continuous improvement from the end of week 9 (continuation phase; 44.8 vs. $23.4 \%$ ). The study was somewhat limited by a small sample size and a bit high dropout rate of $28 \%$ (maybe of treatment-resistant patients?). The sustained remission rates are impressive, though. Results of this study together with results of other discussed studies [28] suggest that creative augmentation of antidepressants or psychotherapy with various thera- 
peutic modalities (psychotherapy, chronotherapeutics, other medications) is a way to go in treating patients with treatment-resistant depression. (No funding for this study is declared, authors claim no conflict of interest.)

\section{Disclosure Statement}

The author declares no conflict of interest.

\section{References}

1 Balon R: Clinical factor 2010. Psychother Psychosom 2011;80:195-198.

2 Balon R: Clinical factor 2011. Psychother Psychosom 2012;81:199-205.

3 Balon R: Clinical factor 2012. Psychother Psychosom 2013;82:204-212.

4 Balon R: Clinical factor 2013. Psychother Psychosom 2014;83:330-340.

5 Balon R: Clinical factor 2014. Psychother Psychosom 2015;84:330-338.

6 Fava GA: The clinical factor. Psychother Psychosom 2011;80:1-3.

7 Giordano GN, Ohlsson H, Sundquist K, Sundquist J, Kendler KS: The association between cannabis abuse and subsequent schizophrenia. Psychol Med 2015;45:407-414.

8 Zorrilla I, Aguado J, Haro JM, Barbeito S, Lopez Zurbano S, Ortiz A, Lopez P, GonzalesPinto A: Cannabis and bipolar disorder: does quitting cannabis use during manic/mixed episode improve clinical/functional outcomes? Acta Psychiatr Scand 2015;131:100110.

9 Wilkinson ST, Stefanovics E, Rosenheck RA: Marijuana use is associated with worse outcomes in symptom severity and violent behavior in patients with posttraumatic stress disorder. J Clin Psychiatry 2015;76:11741180.

10 Freeman TP, Winstock AR: Examining the profile of high-potency cannabis and its association with severity of cannabis dependence. Psychol Med 2015;45:3181-3189.

11 Dodge KA, Bierman KL, Coie JD, Greenberg MT, Lochman JE, McMahon RJ, Pinderhughes EE; Conduct Problems Prevention Research Group: Impact of early intervention on psychopathology, crime, and well-being at age 25. Am J Psychiatry 2015;172:59-70.

12 Wilksch SM, Paxton SJ, Byrne SM, Austin SB, Mclean SA, Thompson KM, Dorairaj K, Wade TD: Prevention across the spectrum: a randomized controlled trial of three programs to reduce risk factors for both eating disorders and obesity. Psychol Med 2015;45: 1811-1823.

13 Ginsburg GS, Drake KL, Tein J-Y, Teetsel R, Riddle MA: Preventing onset of anxiety disorders in offspring of anxious parents: a randomized controlled trial of a family-based intervention. Am J Psychiatry 2015;172:12071214.

14 Ising HK, Smit F, Veling W, Rietdijk J, Dragt S, Klaassen RMC, Savelsberg NSP, Boonstra $\mathrm{N}$, Nieman DH, Linszen DH, Wunderink L, van der Gaag M: Cost-effectiveness of pre- venting first-episode psychosis in ultra-highrisk subjects: multi-centre randomized controlled trial. Psychol Med 2015;45:1435-1446.

15 Imamura K, Kawakami N, Furukawa TA, Matsuyama Y, Shimazu A, Umanodan R, Kawakami S, Kasai K: Does Internet-based cognitive behavioral therapy (iCBT) prevent major depressive episode for workers? A 12-month follow-up of a randomized controlled trial. Psychol Med 2015;45:1907-1917.

16 Imamura K, Kawakami N, Furukawa TA, Matsuyama Y, Shimazu A, Umanodan R, Kawakami S, Kasai K: Effects of an InternetBased Cognitive Behavioral Therapy (iCBT) program in manga format on improving subthreshold depressive symptoms among healthy workers: a randomized controlled trial. PLoS One 2014;9:e97167.

17 Guille C, Zhao Z, Krystal J, Nichols B, Brady K, Sen S: Web-based cognitive behavioral therapy intervention for the prevention of suicidal ideation in medical interns. A randomized clinical trial. JAMA Psychiatry 2015; 72:1192-1198.

18 Pratt D, Tarrier N, Dunn G, Awenat Y, Shaw J, Ulph F, Gooding F: Cognitive-behavioural suicide prevention for male prisoners: a pilot randomized controlled trial. Psychol Med 2015;45:3441-3451.

19 Reynolds CF III: Preventing suicidal ideation in medical interns. JAMA Psychiatry 2015;72: 1169-1170.

20 Berle D, Starcevic V, Milicevic D, Hannan A, Dale E, Brakoulias V, Viswasam K: Do patients prefer face-to-face or Internet-based therapy? Psychother Psychosom 2015;84:6162.

21 Kok G, Burger H, Riper H, Cuijpers P, Dekker J, van Marwijk H, Smit F, Beck A, Bockting $\mathrm{CLH}$ : The three-month effect of mobile Internet-based cognitive therapy of the course of depressive symptoms in remitted recurrently depressed patients: results of a randomized controlled trial. Psychother Psychosom 2015; 84:90-99.

22 Van den Berg N, Grabe H-J, Baumeister SE, Freyberger HJ, Hoffmann W: A telephoneand text message-based telemedicine concept for patients with mental health disorders: results of a randomized controlled trial. Psychother Psychosom 2015;84:82-89.

23 Ngai F-W, Wong PW-C, Leung K-Y, Chau $\mathrm{P}-\mathrm{H}$, Chung K-F: The effect of telephonebased cognitive-behavioral therapy on postnatal depression: a randomized controlled trial. Psychother Psychosom 2015;84:294-303.
24 Brenes GA Danhauer SC, Lyles MF, Hogan PE, Miller ME: Telephone-delivered cognitive behavioral therapy and telephone-delivered nondirective supportive therapy for rural older adults with generalized anxiety disorder. A randomized clinical trial. JAMA Psychiatry 2015;72:1012-1020.

25 Faurholt-Jepsen M, Frost M, Ritz C, Christensen EM, Jacoby AS, Mikkelsen RL, Knorr U, Bardram JE, Vinberg M, Kessing LV: Daily electronic self-monitoring in bipolar disorder using smartphones - the MONARCA I trial: a randomized, placebo controlled, singleblind, parallel group trial. Psychol Med 2015; 45:2691-2704.

26 Kuyken W, Hayes R, Barrett B, Byng R, Dalgleish T, Kessler D, Lewis G, Watkins E, Brejcha C, Cardy J, Causley A, Cowderoy S, Evans A, Gradinger F, Kaur S, Lanham P, Morant M, Richards J, Shah P, Sutton H, Vicary R, Weaver A, Wilks J, Williams M, Taylor RS, Byford $S$ : Effectiveness and cost-effectiveness of mindfulness-based cognitive therapy compared with maintenance antidepressant treatment in the prevention of depressive relapse or recurrence (PREVENT): a randomized controlled trial. Lancet 2015;386:63-73.

27 Mulder R: Depression relapse: importance of longterm perspective. Lancet 2015;386:10-12.

28 Schramm E, Zobel I, Schoepf D, Fangmeier T, Schnell K, Walter H, Drost S, Schmidt P, Brakenmeier E-L, Berger M, Normann C: Cognitive behavioral analysis system of psychotherapy versus escitalopram in chronic major depression. Psychother Psychosom 2015;84:227-240.

29 Martiny K, Refsgaard E, Lund V, Lunde M, Thougaard B, Lindberg L, Bech P: Maintained superiority of chronotherapeutics vs exercise in a 20-week randomized follow-up trial in major depression. Acta Psychiatr Scand 2015; 131:446-457.

30 Martiny K, Refsgaard E, Lund V, Lunde M, Sorensen L, Thougaard B, Lindberg L, Bech P: The day-to-day acute effect of wake therapy in patients with major depression using the HAMD-D6 as primary outcome measure: results from a randomized controlled trial. PLoS One 2013;8:e67264.

31 Martiny K, Refsgaard E, Lund V, Lunde M, Sorensen L, Thougaard B, Lindberg L, Bech P: A 9-week randomized trial comparing a chronotherapeutic intervention (wake and light therapy) to exercise in major depressive disorder patients treated with duloxetine. J Clin Psychiatry 2012;73:1234-1242. 